\title{
THE SHAPE OF CHARGED DROPS OVER A SOLID SURFACE AND SYMMETRY-BREAKING INSTABILITIES*
}

\author{
M. A. FONTELOS ${ }^{\dagger}$ AND U. KINDELÁN ${ }^{\ddagger}$
}

\begin{abstract}
We study the static shape of charged drops of a conducting fluid placed over a solid substrate, surrounded by a gas, and in absence of gravitational forces. The question can be formulated as a variational problem where a certain energy involving the areas of the solid-liquid interface and of the liquid-gas interface, as well as the electric capacity of the drop, has to be minimized. As a function of two parameters, Young's angle $\theta_{Y}$ and the potential at the drop's surface $V_{0}$, we find the axisymmetric minimizers of the energy and describe their shape. We also discuss the existence of symmetry-breaking bifurcations such that, for given values of $\theta_{Y}$ and $V_{0}$, configurations for which the axial symmetry is lost are energetically more favorable than axially symmetric configurations. We prove the existence of such bifurcations in the limits of very flat and almost spherical equilibrium shapes. All other cases are studied numerically with a boundary integral method. One conclusion of this study is that axisymmetric drops cannot spread indefinitely by introducing sufficient amount of electric charges, but can reach only a limiting (saturation) size, after which the axial symmetry would be lost and finger-like shapes energetically preferred.
\end{abstract}

Key words. electrowetting, symmetry-breaking bifurcations, boundary integral method, variational formulation

AMS subject classifications. 35J50, 65N83, 76D 45

DOI. $10.1137 / 080713707$

1. Introduction. The determination of the stationary shapes of liquid drops surrounded by a vapor phase and in contact with a solid surface is an old problem both in fluid mechanics and in the theory of partial differential equations (see [7] and the references therein). The problem can be posed, since Gauss, in a variational setting consisting of obtaining the configurations of a given mass of fluid that minimize (or in general make extremal) an energy defined by

$$
E=\gamma_{l v} A_{l v}-\left(\gamma_{s v}-\gamma_{s l}\right) A_{s l}+E_{F},
$$

where $\gamma_{l v}, \gamma_{s v}$, and $\gamma_{s l}$ denote the liquid-vapor, solid-vapor, and solid-liquid surface tensions, respectively; $A_{l v}$ and $A_{s l}$ denote the area of the liquid-vapor and solid-liquid interfaces, respectively (see Figure 1.1). $E_{F}$ is the contribution of external forces to the total energy. If the drop is affected by gravity, then $E_{F}=\int_{\Omega} \mathbf{g} \cdot \mathbf{x} d x$, where $\mathbf{g}$ is the gravitational force and $\Omega$ the domain occupied by the fluid. In absence of external forces, the configurations that minimize the energy (1.1) are spherical caps such that the contact angle $\theta_{Y}$, called Young's angle, between the liquid-vapor and solid-liquid interfaces satisfies

$$
\cos \theta_{Y}=\frac{\gamma_{s v}-\gamma_{s l}}{\gamma_{l v}}
$$

When the volume of fluid under consideration is sufficiently small, the contribution of gravitational forces to the energy is negligible in comparison with interfacial

\footnotetext{
* Received by the editors January 18, 2008; accepted for publication (in revised form) May 30, 2008; published electronically August 27, 2008.

http://www.siam.org/journals/siap/69-1/71370.html

${ }^{\dagger}$ Instituto de Ciencias Matemáticas (ICMAT, CSIC-UAM-UCM-UC3M), C/ Serrano 123, 28006 Madrid, Spain (marco.fontelos@uam.es).

¥Departamento de Matemática Aplicada y Métodos Informáticos, Universidad Politécnica de Madrid, C/ Ríos Rosas 21, 28003 Madrid, Spain (ultano.kindelan@upm.es).
}

126 